\title{
HOLOCENE MARINE INGRESSIONS IN THE COASTAL ZONE OF THE POMERANIAN BAY BASED ON RADIOCARBON ASSAYS
}

\author{
RYSZARD K. BORÓWKA ${ }^{1}$ and BERNARD CEDRO ${ }^{1}$ \\ ${ }^{I}$ Geology and Palaeogeography Unit, Faculty of Geosciences, University of Szczecin, \\ Mickiewicza 18, 70383 Szczecin, Poland
}

Received 20 May 2010

Accepted 16 September 2010

\begin{abstract}
In recent years, a team at the Geology and Paleogeography Unit, Marine Sciences Institute, University of Szczecin, has been performing geological, geochronological and paleogeographic surveys in (i) the Szczecin Lagoon and Świna Gate Sandbar and (ii) the estuary section of the Rega river valley near Mrzeżyno. These studies have helped to examine and identify not only the distribution of fossil marine sediments but also their lithological and sedimentological characteristics. The age of marine ingressions and regressions in the coastal zone of the Pomeranian Bay were determined using approximately 170 radiocarbon assays.

It was found that the marine ingression associated with the 'Littorina transgression' was not synchronous at these two areas. It started earlier in the Mrzeżyno area, ca. 8300-8200 cal BP. In that first phase, marine sediments developed as tightly packed sands containing a few fragments of shells. In several profiles, these deposits were separated from the bottom and top with layers of peat, thus allowing an indirect determination of their age. The next phase of ingression began about $7300 \mathrm{cal}$ BP. These younger marine sediments already contained quite numerous shells of marine malacofauna, especially Cardium glaucum, often found in a life position.

In the area of the Szczecin Lagoon and Swina Gate Sandbar, the oldest marine ingression started as early as about 7350 cal BP. The quite clear trace is a considerably thicker series of marine sands with numerous sea shells representing marine and brackish-marine environments, including Cardium glaucum shells in a life position, which made it possible to identify the age and the rate of accumulation of marine sands.
\end{abstract}

Keywords: marine ingression, Baltic sea level changes, Holocene, Pomeranian Bay.

\section{INTRODUCTION}

The age of marine ingressions on the Polish Baltic coast has been discussed for a long time. Usually, the discussions have not been based on age determinations of marine sediments, but rather used dating of organic matter (peat, gyttja, wood fragments) underlying or covering marine layers.

The aim of this study was to determine the actual age of the marine sand layers documented in a number of

Corresponding author: R. K. Borówka

e-mail: ryszard@univ.szczecin.pl locations in the coastal zone of the Pomeranian Bay (Borówka et al., 1999, 2002, 2005; Cedro, 2003, 2005). A conducive factor was the presence of fossil shells of Cardium glaucum in marine sediments, some of which were preserved in a life position. This mollusc species is particularly poorly resistant to being buried in sediments, due to its very short siphon (Wołowicz, 1991). Therefore, the radiocarbon age of the intact shells found is a de facto age of deposits where they were buried (taking into account the reservoir effect).

An additional factor which enabled higher dating precision is the fact that the mollusc Cardium glaucum has a 
short life, max. 3-4 years (Wołowicz, 1991). Hence the determination of the age of whole shells did not require taking into account the age of the mollusc.

\section{STUDY AREA}

The study was conducted in two test areas located on the Polish coast of the southern Baltic Sea (Fig. 1). The areas included the present area of Szczecin Lagoon $\left(54^{\circ} 48^{\prime} \mathrm{N}, 14^{\circ} 20^{\prime} \mathrm{E}\right)$ and the estuary of the Rega river $\left(54^{\circ} 48^{\prime} \mathrm{N}, 14^{\circ} 20^{\prime} \mathrm{E}\right)$.

Szczecin Lagoon has an area of approximately 665 $\mathrm{km}^{2}$. It is a shallow freshwater body, with a natural depth not exceeding $8.5 \mathrm{~m}$, fed mainly by the Oder and its smaller tributaries: Ücker and Peene. The sediments filling the eastern part of the lagoon (Great Lagoon) are at max. 5 metres thick and lie on fluvial sands on lower lying glaciofluvial gravels and sands (Borówka et al., 2002; 2005). The deposits in stratigraphic order are: late glacial, early and middle holocene peat and gyttjas, sands with marine fauna of Cardium glaucum, Macoma balthica, Mytilus edulis, Hydrobia ventrosa and Hydrobia ulvae, and then algal and silt gyttjas with lacustrine malacofauna (Borówka et al., 2002; 2005).

The Rega river estuary occupies a former portion of the marginal valley, with longitudinal orientation and a general westward decline (Karczewski, 1968). The fossil bottom of the valley is a residual lag shearing interplenivistulian sand and silt sediments, and vistulian tills on the slopes. The deposits of this valley in stratigraphic order are: glaciofluvial sands and gravels, late glacial silts and clays, early and mid-holocene peats and gyttjas, sands with marine fauna from Cardium glaucum, Macoma balthica and Mytilus edulis separated by a layer of peat, in lateral contact with the sands in the north and lacustrine deposits in the south. This sequence is covered by mid- and late-holocene peats and then aeolian sands (Cedro, 2005; 2008).

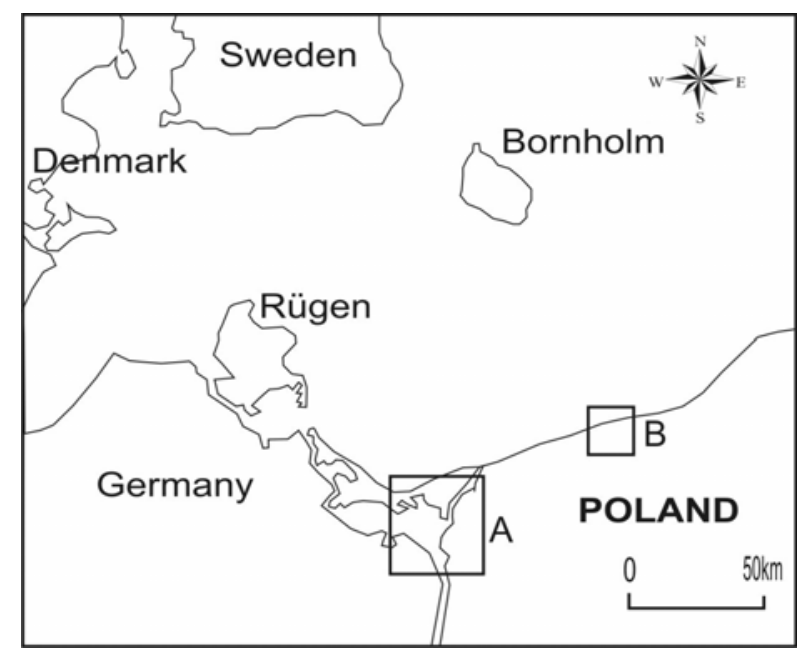

Fig. 1. Study area A - Szczecin Bay , B - Mrzeżyno.

\section{MATERIALS AND METHODS}

The materials of this research were deposits filling the aforementioned sedimentary basins. In Szczecin Lagoon they were identified using 38 vibroprobes reaching about $4 \mathrm{~m}$ below the present-day seabed (Fig. 2). In the Rega estuary 29 test drillings were performed, with depths ranging from 7.5 to 13 meters (Fig. 3).

From the selected vibroprobes and drillings, samples of organic sediments were collected, including Cardium glaucum shells, in order to determine their radiocarbon

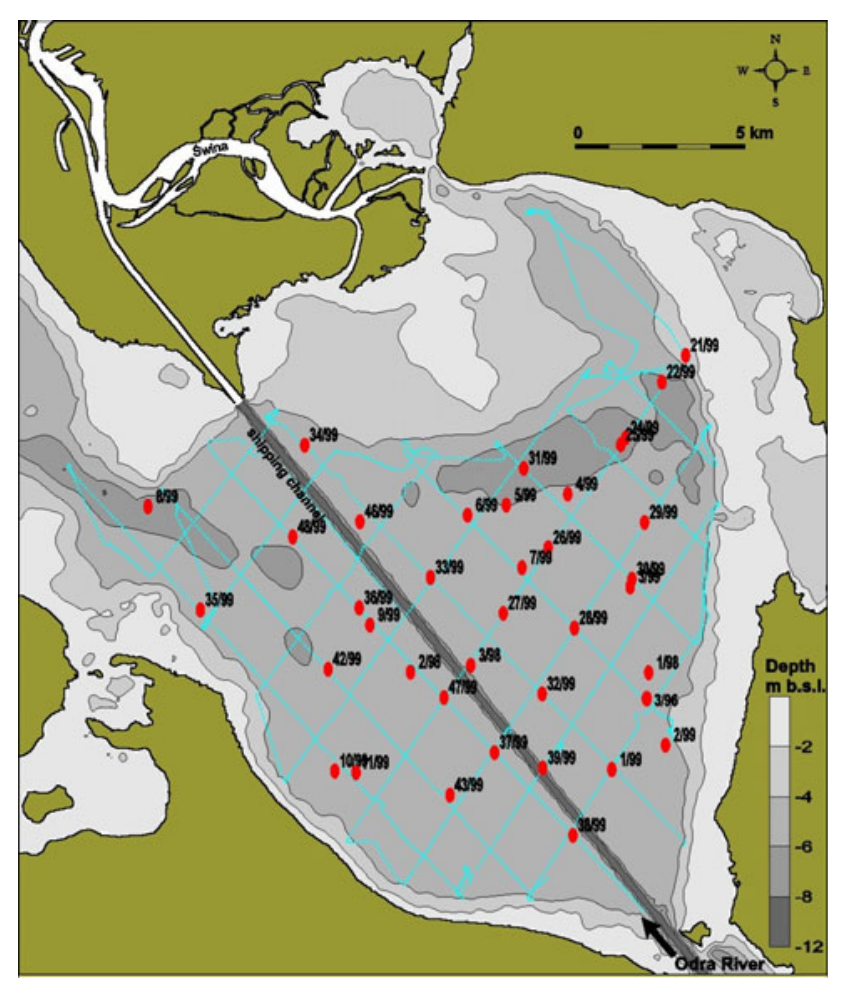

Fig. 2. Location of sediment cores ( $A$ area).

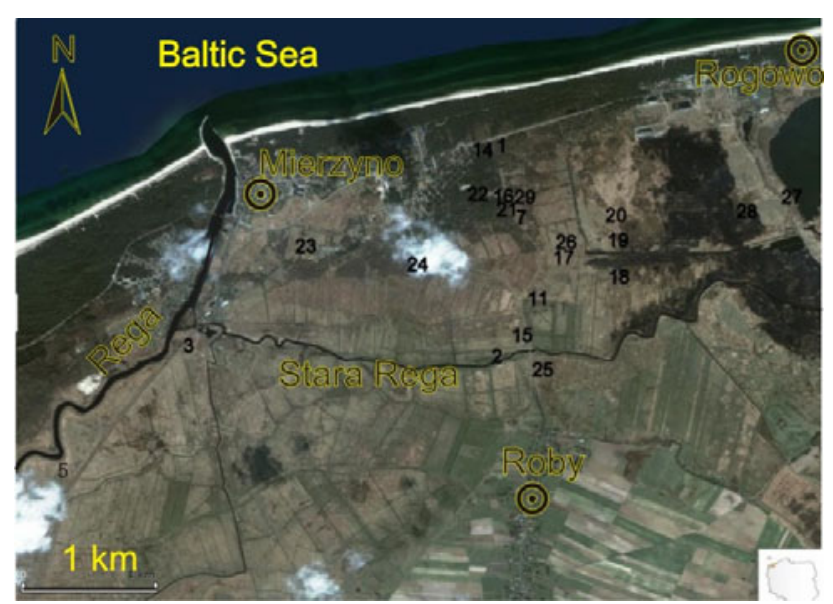

Fig. 3. Location of sediment cores ( $B$ area). 
ages. Gyttja and peat samples were dated at the Radiocarbon Laboratory, Institute of Physics, Gliwice. The age of Cardium glaucum shells found in life position was determined using AMS at Poznań Radiocarbon Laboratory. The samples comprised 118 gyttja and peat samples, 7 wood samples and 70 samples of shells.

The reservoir age of Cardium shells was assumed as 300 years, according to values for the southern Baltic based on the Chrono Marine Reservoir Database (http://intcal.qub.ac.uk/marine/).

Calendar ages were determined using an Internet programme OxCal (https://c14.arch.ox.ac.uk, Bronk Ramsey, 2009) and calibration curve IntCal09 (Reimer et al., 2009) - see Table 1 and 2.

\section{RESULTS}

\section{Szczecin Lagoon}

In the area of Szczecin Lagoon, radiocarbon dating was performed on 56 samples of organic sediments and 67 Cardium glaucum shells. Selected results from nine geological probes are presented in Table 1.

Only those results which are significant for the determination of the age of marine sediments and organic deposits below them have been presented. Results of calibration for all samples are presented as frequency distribution of radiocarbon dates on calendar time scale in Fig. 4.

The ages of organic sediments fall in two groups, i.e., from about 15,000 until $6000 \mathrm{cal}$ BP and after $3500 \mathrm{cal}$ BP. The age of Cardium glaucum shells, showing the period of accumulation of marine sediments in the present day Szczecin Lagoon, ranges from about 7200 to $3800 \mathrm{cal} \mathrm{BP}$ (Table 1).

Marine sediments which developed as fine and medium sands at the bottom and silts at the top, are located mainly on limnic and swamp sediments, especially in the central part of the Great Lagoon, and on fluvial deposits in the western parts. Their base occurs at depth of $-10 \mathrm{~m}$ to $-6.5 \mathrm{~m}$ amsl with a thickness rarely exceeding $1.5 \mathrm{~m}$.

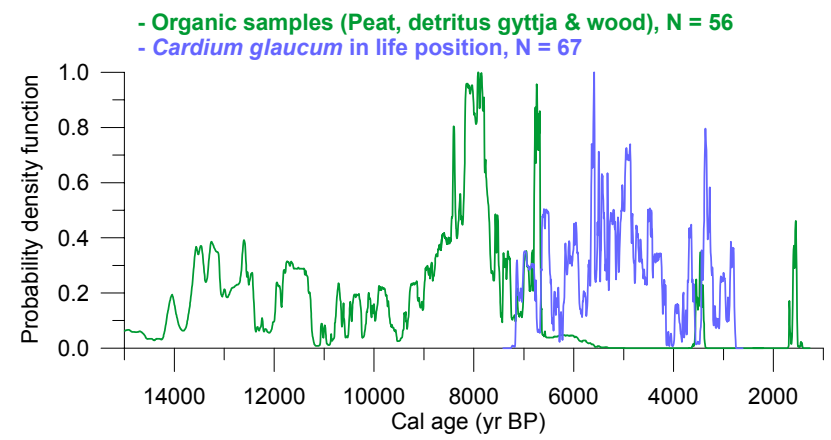

Fig. 4. Frequency distributions of radiocarbon dates of organic and shell samples on calendar time scale. The curves were constructed by summing up probability distributions of individual dates (Option "Sum" in OxCal programme)(A area).
Based on the results of radiocarbon age determinations presented in the Table 1 and the example of a profile (Fig. 5), it can be assumed that in the area of Szczecin Lagoon the sea ingression began at the earliest around 7360 years cal BP, and at the latest about 7000 years cal BP. This is evidenced by the oldest mollusc shells derived from the bottom of the marine sediments (ZSW 78/93 - see Table 1), and is further supported by the results of age determinations of the youngest layers of peat and organic mud under the marine sediments (ZS 28/57 - see table). The sea lagoon lasted until about 3800 years cal BP. Based on data from profile 35/99 (Fig. 5) and other locations, it appears that in this relatively long period there was a continuity of marine sedimentation.

\section{Area of the Rega river estuary}

In the estuary of the Rega River, radiocarbon determinations were performed for 67 samples (Fig. 6) of organic sediment samples and 3 shell samples from 29 boreholes. Selected results from 8 boreholes are presented in Table 2.

The age of organic sediments is grouped in several classes, i.e. from 14,000 to 12,000 years cal BP, from 11,000 to $7500 \mathrm{cal} \mathrm{BP}$ to, from 7000 to $4500 \mathrm{cal} \mathrm{BP}$ and from 3000 to $1000 \mathrm{cal} \mathrm{BP}$.

The dating results presented in the Table 2 and the example of a profile (Fig. 7) show that in the vicinity of Mrzeżyno (estuary of the Rega River), the earlier phase of sea ingression occurred between 8360 cal BP and 8180 cal BP. That phase of ingression is represented by heavily packed marine sand deposits containing a few fragments of shells. They can be found at the ordinate $-7 \mathrm{~m}$ amsl. These deposits are both underlying and covering layers of peat. The peats underlying the deposits with the aforementioned age were found in three bores/drillings, and peat covering the deposits in seven drillings. The younger layer of peat was accumulated in the period between 8180 cal BP and $7640 \mathrm{cal} \mathrm{BP}$. On the layer at the ordinate
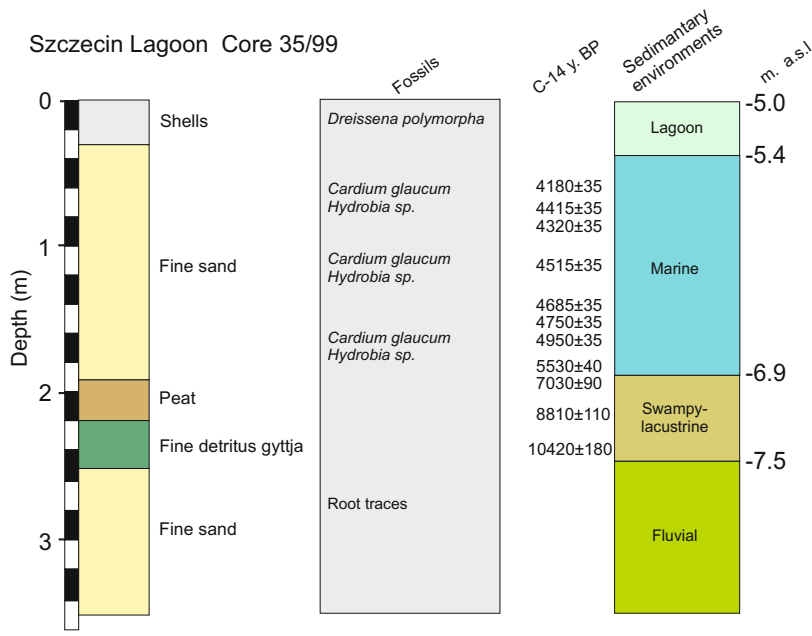

Fig. 5. Sediment cores. 
Table 1. Dating and calibration results.

\begin{tabular}{|c|c|c|c|c|c|c|c|c|c|c|}
\hline No & $\begin{array}{c}\text { Sample } \\
\text { name }\end{array}$ & $\begin{array}{c}\begin{array}{c}\text { No. of } \\
\text { the } \\
\text { location } \\
\text { (core) }\end{array}\end{array}$ & $\begin{array}{c}\text { Type of } \\
\text { dated } \\
\text { sediment }\end{array}$ & $\begin{array}{c}\text { Depth } \\
\text { (meters } \\
\text { below the } \\
\text { terrain or } \\
\text { sea bottom } \\
\text { level) }\end{array}$ & $\begin{array}{l}\text { Ordinate } \\
\text { ( } \mathrm{m} \text { ams } \mathrm{l})\end{array}$ & Latitude & Longitude & $\begin{array}{l}\text { Radiocarbon } \\
\text { age (years } \\
{ }^{14} \mathrm{C} \mathrm{BP} \text { ) }\end{array}$ & $\begin{array}{c}68.2 \% \text { confidence intervals Cal } \\
\text { agerange (year BP) }\end{array}$ & Lab ID \\
\hline 1 & 2 & 3 & 4 & 5 & 6 & 7 & 8 & 9 & 10 & 11 \\
\hline 1 & ZS 9 & $9 / 96$ & organic silt & $4.90-5.00$ & -10 & $53^{\circ} 44^{\prime} 31^{\prime \prime}$ & $14^{\circ} 30^{\prime} 32^{\prime \prime}$ & $7250 \pm 130$ & $7940-8200(68.2 \%)$ & Gd-15092 \\
\hline 2 & ZS 26/56 & $26 / 99$ & $\begin{array}{l}\text { detritus } \\
\text { gyttja }\end{array}$ & $2.75-2.80$ & -8.75 & $53^{\circ} 46^{\prime} 23^{\prime \prime}$ & $14^{\circ} 28^{\prime} 01^{\prime \prime}$ & $7240 \pm 120$ & $7950-8180(68.2 \%)$ & Gd-15103 \\
\hline 3 & ZS 28/57 & $28 / 99$ & organic silt & $2.80-285$ & -8.57 & $53^{\circ} 45^{\prime} 11^{\prime \prime}$ & $14^{\circ} 28^{\prime} 41^{\prime \prime}$ & $6230 \pm 250$ & $6800-7450(68.2 \%)$ & Gd-16027 \\
\hline 4 & ZS 29/26 & $29 / 99$ & organic silt & $1.24-1.30$ & -7.02 & $53^{\circ} 46^{\prime} 48^{\prime \prime}$ & $14^{\circ} 30^{\prime} 25^{\prime \prime}$ & $7050 \pm 90$ & $7790-7970(68.2 \%)$ & Gd-12241 \\
\hline 5 & ZS 35/12 & $35 / 99$ & $\begin{array}{l}\text { Cardium } \\
\text { glaucum }\end{array}$ & $0.52-0.56$ & -5.57 & $53^{\circ} 45^{\prime} 29^{\prime \prime}$ & $14^{\circ} 19^{\prime} 26^{\prime \prime}$ & $4180 \pm 35$ & $\begin{array}{l}4800-4830(14.3 \%) \\
4690-4770(38.4 \%) \\
4630-4680(15.5 \%)\end{array}$ & Poz-12391 \\
\hline 6 & ZS 35/14 & $35 / 99$ & $\begin{array}{l}\text { Cardium } \\
\text { glaucum }\end{array}$ & $0.60-0.65$ & -5.66 & $53^{\circ} 45^{\prime} 29^{\prime \prime}$ & $14^{\circ} 19^{\prime} 26^{\prime \prime}$ & $4415 \pm 35$ & $\begin{array}{l}4950-5050(47.6 \%) \\
4880-4940(20.6 \%)\end{array}$ & Poz-12392 \\
\hline 7 & ZS 35/18 & $35 / 99$ & $\begin{array}{l}\text { Cardium } \\
\text { glaucum }\end{array}$ & $0.80-0.85$ & -5.86 & $53^{\circ} 45^{\prime} 29^{\prime \prime}$ & $14^{\circ} 19^{\prime} 26^{\prime \prime}$ & $4320 \pm 35$ & $\begin{array}{l}4930-4960(19.1 \%) \\
4840-4890(49.1 \%)\end{array}$ & Poz-12394 \\
\hline 8 & ZS 35/25 & $35 / 99$ & $\begin{array}{l}\text { Cardium } \\
\text { glaucum }\end{array}$ & $1.10-1.15$ & -6.16 & $53^{\circ} 45^{\prime} 29^{\prime \prime}$ & $14^{\circ} 19^{\prime} 26^{\prime \prime}$ & $4515 \pm 35$ & $\begin{array}{c}5270-5300(11.9 \%) \\
5210-5230(2.5 \%) \\
5060-5190(53.9 \%)\end{array}$ & Poz-12395 \\
\hline 9 & ZS 35/30 & $35 / 99$ & $\begin{array}{l}\text { Cardium } \\
\text { glaucum }\end{array}$ & $1.35-1.40$ & -6.41 & $53^{\circ} 45^{\prime} 29^{\prime \prime}$ & $14^{\circ} 19^{\prime} 26^{\prime \prime}$ & $4685 \pm 35$ & $\begin{array}{l}5440-5470(15.4 \%) \\
5320-5420(52.8 \%)\end{array}$ & Poz-12396 \\
\hline 10 & ZS $35 / 32$ & $35 / 99$ & $\begin{array}{l}\text { Cardium } \\
\text { glaucum }\end{array}$ & $1.45-1.50$ & -6.51 & $53^{\circ} 45^{\prime} 29^{\prime \prime}$ & $14^{\circ} 19^{\prime} 26^{\prime \prime}$ & $4750 \pm 35$ & $\begin{array}{l}5500-5590(54.2 \%) \\
5460-5490(14.0 \%)\end{array}$ & Poz-12397 \\
\hline 11 & ZS 35/34 & $35 / 99$ & $\begin{array}{l}\text { Cardium } \\
\text { glaucum }\end{array}$ & $1.55-1.60$ & -6.61 & $53^{\circ} 45^{\prime} 29^{\prime \prime}$ & $14^{\circ} 19^{\prime} 26^{\prime \prime}$ & \pm 35 & $\begin{array}{l}5640-5720(62.4 \%) \\
5610-5630(5.8 \%)\end{array}$ & Poz-12398 \\
\hline 12 & ZS 35/39 & $35 / 99$ & $\begin{array}{l}\text { Cardium } \\
\text { glaucum }\end{array}$ & $1.80-1.85$ & -6.86 & $53^{\circ} 45^{\prime} 29^{\prime \prime}$ & $14^{\circ} 19^{\prime} 26^{\prime \prime}$ & $5530 \pm 40$ & $\begin{array}{l}6360-6400(22.0 \%) \\
6280-6350(46.3 \%) \\
\end{array}$ & Poz-12399 \\
\hline 13 & ZS 35/42 & $35 / 99$ & peat & $1.92-2.00$ & -7.01 & $53^{\circ} 45^{\prime} 29^{\prime \prime}$ & $14^{\circ} 19^{\prime} 26^{\prime \prime}$ & $7030 \pm 90$ & $7760-7960(68.2 \%)$ & Gd-12223 \\
\hline 14 & ZS 35/46 & $35 / 99$ & peat & $2.15-2.20$ & -7.21 & $53^{\circ} 45^{\prime} 29^{\prime \prime}$ & $14^{\circ} 19^{\prime} 26^{\prime \prime}$ & $8810 \pm 110$ & $\begin{array}{c}10060-10150(14.0 \%) \\
9980-10050(6.5 \%) \\
9680-9950(47.6 \%)\end{array}$ & Gd-12224 \\
\hline 15 & ZS 35/50 & $35 / 99$ & $\begin{array}{l}\text { detritus } \\
\text { gyttja }\end{array}$ & $2.35-2.40$ & -7.41 & $53^{\circ} 45^{\prime} 29^{\prime \prime}$ & $14^{\circ} 19^{\prime} 26^{\prime \prime}$ & $10420 \pm 180$ & $12040-12560(68.2 \%)$ & Gd-15090 \\
\hline 16 & ZS 42/6 & $42 / 99$ & gyttja & $0.30-0.35$ & -6.4 & $53^{\circ} 44^{\prime} 35^{\prime \prime}$ & $14^{\circ} 22^{\prime} 37^{\prime \prime}$ & $1670 \pm 30$ & $1530-1610(68.2 \%)$ & Poz-796 \\
\hline 17 & ZS 42/12 & $42 / 99$ & gyttja & $0.65-0.70$ & -6.75 & $53^{\circ} 44^{\prime} 35^{\prime \prime}$ & $14^{\circ} 22^{\prime} 37^{\prime \prime}$ & $3250 \pm 40$ & $\begin{array}{c}3520-3560(17.1 \%) \\
3500-3510(2.1 \%) \\
3440-3490(35.8 \%) \\
3400-3430(13.2 \%)\end{array}$ & Poz-816 \\
\hline 18 & ZS 42/20 & $42 / 99$ & $\begin{array}{l}\text { Cardium } \\
\text { glaucum }\end{array}$ & $1.00-1.05$ & -7.1 & $53^{\circ} 44^{\prime} 35^{\prime \prime}$ & $14^{\circ} 22^{\prime} 37^{\prime \prime}$ & $4210 \pm 35$ & $\begin{array}{c}4810-4840(23.8 \%) \\
4700-4760(37.1 \%) \\
4650-4670(7.2 \%)\end{array}$ & Poz-705 \\
\hline 19 & ZS 42/21 & $42 / 99$ & $\begin{array}{l}\text { Cardium } \\
\text { glaucum }\end{array}$ & $1.05-1.10$ & -7.15 & $53^{\circ} 44^{\prime} 35^{\prime \prime}$ & $14^{\circ} 22^{\prime} 37^{\prime \prime}$ & $4510 \pm 35$ & $\begin{array}{c}5260-5290(11.0 \%) \\
5210-5250(4.5 \%) \\
5050-5190(52.7 \%)\end{array}$ & Poz-706 \\
\hline 20 & ZS 42/24 & $42 / 99$ & $\begin{array}{l}\text { Cardium } \\
\text { glaucum }\end{array}$ & $1.20-1.25$ & -7.3 & $53^{\circ} 44^{\prime} 35^{\prime \prime}$ & $14^{\circ} 22^{\prime} 37^{\prime \prime}$ & $5140 \pm 40$ & $\begin{array}{l}5880-5940(45.4 \%) \\
5760-5810(22.8 \%)\end{array}$ & Poz-703 \\
\hline 21 & ZS 42/28 & $42 / 99$ & $\begin{array}{l}\text { Cardium } \\
\text { glaucum }\end{array}$ & $1.40-1.45$ & -7.5 & $53^{\circ} 44^{\prime} 35^{\prime \prime}$ & $14^{\circ} 22^{\prime} 37^{\prime \prime}$ & $6430 \pm 45$ & $7320-7420(68.2 \%)$ & Poz-702 \\
\hline 22 & ZS $42 / 30$ & $42 / 99$ & $\begin{array}{l}\text { detritus } \\
\text { gyttja }\end{array}$ & $1.50-1.55$ & -7.6 & $53^{\circ} 44^{\prime} 35^{\prime \prime}$ & $14^{\circ} 22^{\prime} 37^{\prime \prime}$ & $6850 \pm 90$ & $7600-7790(68.2 \%)$ & Gd-12217 \\
\hline 23 & ZS 42/36 & $42 / 99$ & $\begin{array}{l}\text { detritus } \\
\text { gyttja }\end{array}$ & $1.80-1.86$ & -7.91 & $53^{\circ} 44^{\prime} 35^{\prime \prime}$ & $14^{\circ} 22^{\prime} 37^{\prime \prime}$ & $7320 \pm 80$ & $8020-8200(68.2 \%)$ & Gd-12222 \\
\hline 24 & ZS 42/41 & $42 / 99$ & peat & $2.00-2.03$ & -8.08 & $53^{\circ} 44^{\prime} 35^{\prime \prime}$ & $14^{\circ} 22^{\prime} 37^{\prime \prime}$ & $10030 \pm 120$ & $11310-11760(68.2 \%)$ & Gd-12214 \\
\hline 25 & ZS 42/44 & $42 / 99$ & peat & $2.10-2.14$ & -8.19 & $53^{\circ} 44^{\prime} 35^{\prime \prime}$ & $14^{\circ} 22^{\prime} 37^{\prime \prime}$ & $11450 \pm 140$ & $13160-13440(68.2 \%)$ & Gd-12220 \\
\hline 26 & $\begin{array}{l}\text { ZSW } \\
42 / 173\end{array}$ & $\begin{array}{c}\text { ZSW- } \\
042\end{array}$ & $\begin{array}{l}\text { Cardium } \\
\text { glaucum }\end{array}$ & 1.73 & -7.13 & $53^{\circ} 48^{\prime} 33^{\prime \prime}$ & $14^{\circ} 28^{\prime} 18^{\prime \prime}$ & $3735 \pm 35$ & $\begin{array}{l}4070-4150(43.2 \%) \\
3990-4040(25.0 \%)\end{array}$ & Poz-28288 \\
\hline 27 & \begin{tabular}{|l|} 
ZSW \\
$42 / 232$ \\
\end{tabular} & $\begin{array}{c}\text { ZSW- } \\
042\end{array}$ & $\begin{array}{l}\text { Cardium } \\
\text { glaucum }\end{array}$ & 2.32 & -7.72 & $53^{\circ} 48^{\prime} 33^{\prime \prime}$ & $14^{\circ} 28^{\prime} 18^{\prime \prime}$ & $6270 \pm 40$ & $7170-7250(68.2 \%)$ & Poz-28295 \\
\hline 28 & \begin{tabular}{|l|} 
ZSW \\
$57 / 131$ \\
\end{tabular} & $\begin{array}{l}\text { ZSW- } \\
057\end{array}$ & $\begin{array}{l}\text { Cardium } \\
\text { glaucum }\end{array}$ & 1.31 & -7.31 & $53^{\circ} 47^{\prime} 35^{\prime \prime}$ & $14^{\circ} 24^{\prime} 18^{\prime \prime}$ & $3920 \pm 35$ & $4290-4420(68.2 \%)$ & Poz-28297 \\
\hline 29 & $\begin{array}{l}\text { ZSW } \\
57 / 237 \\
\end{array}$ & $\begin{array}{l}\text { ZSW- } \\
057\end{array}$ & $\begin{array}{l}\text { Cardium } \\
\text { glaucum }\end{array}$ & 2.37 & -8.37 & $53^{\circ} 47^{\prime} 35^{\prime \prime}$ & $14^{\circ} 24^{\prime} 18^{\prime \prime}$ & $6410 \pm 40$ & $\begin{array}{l}7350-7420(45.8 \%) \\
7300-7340(22.4 \%)\end{array}$ & Poz-28304 \\
\hline 30 & $\begin{array}{l}\text { ZSW } \\
78 / 93\end{array}$ & $\begin{array}{l}\text { ZSW- } \\
078\end{array}$ & $\begin{array}{l}\text { Cardium } \\
\text { glaucum }\end{array}$ & 0.93 & -6.83 & $53^{\circ} 46^{\prime} 01^{\prime \prime}$ & $14^{\circ} 25^{\prime} 50^{\prime \prime}$ & $6460 \pm 40$ & $\begin{array}{l}7410-7430(12.0 \%) \\
7320-7400(56.2 \%)\end{array}$ & Poz-28312 \\
\hline
\end{tabular}


Table 2. Dating and calibration results.

\begin{tabular}{|c|c|c|c|c|c|c|c|c|c|c|}
\hline No & $\begin{array}{c}\text { Sample } \\
\text { name }\end{array}$ & \begin{tabular}{|c|}
$\begin{array}{c}\text { No. of } \\
\text { the } \\
\text { location } \\
\text { (core) }\end{array}$ \\
\end{tabular} & $\begin{array}{c}\text { Type of } \\
\text { dated } \\
\text { sediment }\end{array}$ & $\begin{array}{c}\text { Depth } \\
\text { (meters } \\
\text { below the } \\
\text { terrain or } \\
\text { sea } \\
\text { bottom } \\
\text { level) }\end{array}$ & \begin{tabular}{|l|} 
Ordinate \\
(m amsI)
\end{tabular} & Latitude & Longitude & $\begin{array}{c}\text { Radiocarbon } \\
\text { age (years } \\
{ }^{14} \mathrm{C} \mathrm{BP} \text { ) }\end{array}$ & $\begin{array}{c}68.2 \% \text { confidence intervals Cal ager- } \\
\text { ange (year BP) }\end{array}$ & Lab ID \\
\hline 1 & 2 & 3 & 4 & 5 & 6 & 7 & 8 & 9 & 10 & 11 \\
\hline 1 & $\operatorname{Trz} 7 / 16$ & Trz7 & peat & $2.75-2.80$ & -1.3 & $54^{\circ} 8^{\prime} 37^{\prime \prime}$ & $15^{\circ} 18^{\prime} 54^{\prime \prime}$ & $5100 \pm 140$ & $\begin{array}{c}5962-5990(4.9 \%) \\
5660-5952(63.3 \%)\end{array}$ & Gd-15660 \\
\hline 2 & Trz7/28 & Trz7 & wood & \begin{tabular}{|l|}
$4.70-4.75$ \\
\end{tabular} & -3.2 & $54^{\circ} 8^{\prime} 37^{\prime \prime}$ & $15^{\circ} 18^{\prime} 54^{\prime \prime}$ & $7330 \pm 55$ & $8046-8185(68.2 \%)$ & Gd-15658 \\
\hline 3 & Trz7/38 & Trz7 & peat & $6.20-6.30$ & -4.7 & $54^{\circ} 8^{\prime} 37^{\prime \prime}$ & $15^{\circ} 18^{\prime} 54^{\prime \prime}$ & $7160 \pm 50$ & $7941-8014(68.2 \%)$ & Poz33390 \\
\hline 4 & $\operatorname{Trz} 7 / 46$ & Trz7 & peat & $7.70-7.75$ & -6.2 & $54^{\circ} 8^{\prime} 37^{\prime \prime}$ & $15^{\circ} 18^{\prime} 54^{\prime \prime}$ & $7680 \pm 40$ & $\begin{array}{l}8494-8516(16.8 \%) \\
8417-8480(51.4 \%)\end{array}$ & Gd-12641 \\
\hline 5 & Trz7/74 & Trz7 & peat & $\begin{array}{l}10.95- \\
11.00\end{array}$ & -9.5 & $54^{\circ} 8^{\prime} 37^{\prime \prime}$ & $15^{\circ} 18^{\prime} 54^{\prime \prime}$ & $10390 \pm 220$ & $\begin{array}{c}11960-12572(64.7 \%) \\
11830-11875(3.5 \%)\end{array}$ & Gd-15670 \\
\hline 6 & Trz19/72 & Trz19 & $\begin{array}{l}\text { detritus } \\
\text { gyttja }\end{array}$ & $7.40-7.50$ & -6.4 & $54^{\circ} 8^{\prime} 23^{\prime \prime}$ & $15^{\circ} 19^{\prime} 26^{\prime \prime}$ & $14340 \pm 170$ & $17170-17672(68.2 \%)$ & Gd-12833 \\
\hline 7 & Trz20/31 & Trz20 & peat & $3.50-3.55$ & -2 & $54^{\circ} 8^{\prime} 28^{\prime \prime}$ & $15^{\circ} 19^{\prime} 25^{\prime \prime}$ & $5900 \pm 70$ & $\begin{array}{l}6820-6824(0.9 \%) \\
6639-6797(67.3 \%)\end{array}$ & Gd-15910 \\
\hline 8 & Trz20/54 & Trz20 & peat & $5.75-5.85$ & -4.3 & $54^{\circ} 8^{\prime} 28^{\prime \prime}$ & $15^{\circ} 19^{\prime} 25^{\prime \prime}$ & $7170 \pm 75$ & $\begin{array}{c}8142-8151(2.0 \%) \\
8124-8128(0.8 \%) \\
8095-8104(2.0 \%) \\
7930-8050(59.2 \%) \\
7876-7892(4.1 \%)\end{array}$ & Gd-12835 \\
\hline 9 & Trz21/34 & Trz21 & $\begin{array}{l}\text { Cardium } \\
\text { glaucum }\end{array}$ & $5.70-5.80$ & -3.2 & $54^{\circ} 8^{\prime} 35^{\prime \prime}$ & $15^{\circ} 18^{\prime} 53^{\prime \prime}$ & $6540 \pm 40$ & $7424-7479(68.2 \%)$ & Poz 20538 \\
\hline 10 & Trz21/48 & Trz21 & peat & $7.20-7.30$ & -5.8 & $54^{\circ} 8^{\prime} 35^{\prime \prime}$ & $15^{\circ} 18^{\prime} 53^{\prime \prime}$ & $7350 \pm 80$ & $\begin{array}{l}8261-8296(10.5 \%) \\
8040-8210(57.7 \%)\end{array}$ & GdS-781 \\
\hline 11 & Trz22/32 & Trz22 & $\begin{array}{l}\text { Cardium } \\
\text { glaucum }\end{array}$ & $6.80-6.85$ & -5.3 & $54^{\circ} 8^{\prime} 34^{\prime \prime}$ & $15^{\circ} 18^{\prime} 43^{\prime \prime}$ & $6740 \pm 40$ & $\begin{array}{c}7640-7651(7.8 \%) \\
7572-7622(60.4 \%)\end{array}$ & Poz 20539 \\
\hline 12 & Trz26/5 & Trz26 & peat & \begin{tabular}{|l|}
$0.50-0.55$ \\
\end{tabular} & 0.50 & $54^{\circ} 8^{\prime} 24^{\prime \prime}$ & $15^{\circ} 19^{\prime} 10^{\prime \prime}$ & $1450 \pm 25$ & $1308-1354(68.2 \%)$ & GdS-787 \\
\hline 13 & Trz26/20 & $\operatorname{Trz} 26$ & peat & $2.35-2.40$ & -1.4 & $54^{\circ} 8^{\prime} 24^{\prime \prime}$ & $15^{\circ} 19^{\prime} 10^{\prime \prime}$ & $4400 \pm 80$ & $\begin{array}{c}5244-5260(3.2 \%) \\
5224-5235(2.2 \%) \\
5188-5214(5.9 \%) \\
4858-5054(56.9 \%) \\
\end{array}$ & GdS-779 \\
\hline 14 & Trz26/48 & Trz26 & $\begin{array}{l}\text { Cardium } \\
\text { glaucum }\end{array}$ & $5.00-5.10$ & -4.1 & $54^{\circ} 8^{\prime} 24^{\prime \prime}$ & $15^{\circ} 19^{\prime} 10^{\prime \prime}$ & $6640 \pm 40$ & $7494-7568(68.2 \%)$ & Poz-33389 \\
\hline 15 & Trz26/50 & Trz26 & peat & $5.15-5.20$ & -4.2 & $54^{\circ} 8^{\prime} 24^{\prime \prime}$ & $15^{\circ} 19^{\prime} 10^{\prime \prime}$ & $6775 \pm 85$ & $\begin{array}{c}7566-7692(66.7 \%) \\
7524-7530(1.5 \%) \\
\end{array}$ & Gd-11983 \\
\hline 16 & Trz26/56 & Trz26 & organic silt & $5.60-5.65$ & -4.6 & $54^{\circ} 8^{\prime} 24^{\prime \prime}$ & $15^{\circ} 19^{\prime} 10^{\prime \prime}$ & $9060 \pm 260$ & $\begin{array}{c}9886-10560(63.4 \%) \\
9866-9876(0.7 \%) \\
9788-9848(4.2 \%) \\
\end{array}$ & Gd-30180 \\
\hline 17 & Trz28/9 & Trz28 & peat & $0.90-0.95$ & -0.1 & $54^{\circ} 8^{\prime} 36^{\prime \prime}$ & $15^{\circ} 20^{\prime} 9^{\prime \prime}$ & $2270 \pm 50$ & $\begin{array}{l}2303-2345(31.1 \%) \\
2180-2240(37.1 \%)\end{array}$ & GdS-780 \\
\hline 18 & Trz28/50 & Trz28 & $\begin{array}{c}\text { detritus } \\
\text { gyttja }\end{array}$ & $5.10-5.20$ & -4.1 & $54^{\circ} 8^{\prime} 36^{\prime \prime}$ & $15^{\circ} 20^{\prime} 9^{\prime \prime}$ & $4680 \pm 80$ & $\begin{array}{l}5546-5575(9.2 \%) \\
5316-5474(59.0 \%) \\
\end{array}$ & Poz-33386 \\
\hline 19 & Trz28/70 & Trz28 & $\begin{array}{c}\text { detritus } \\
\text { gyttja }\end{array}$ & $6.70-6.80$ & -5.7 & $54^{\circ} 8^{\prime} 36^{\prime \prime}$ & $15^{\circ} 20^{\prime} 9^{\prime \prime}$ & $7170 \pm 50$ & $7946-8020(68.2 \%)$ & Poz-33387 \\
\hline 20 & Trz28/82 & Trz28 & peat & $7.60-7.70$ & -6.7 & $54^{\circ} 8^{\prime} 36^{\prime \prime}$ & $15^{\circ} 20^{\prime} 9^{\prime \prime}$ & $6930 \pm 50$ & $\begin{array}{c}7811-7822(5.1 \%) \\
7690-7795(63.1 \%)\end{array}$ & Poz-33388 \\
\hline 21 & Trz28/87 & Trz28 & wood & $7.90-7.95$ & -6.9 & $54^{\circ} 8^{\prime} 36^{\prime \prime}$ & $15^{\circ} 20^{\prime} 9^{\prime \prime}$ & $7370 \pm 70$ & $\begin{array}{c}8156-8315(56.2 \%) \\
8110-8117(1.8 \%) \\
8056-8090(10.2 \%)\end{array}$ & GdS-768 \\
\hline 22 & Trz28/93 & $\operatorname{Trz} 28$ & peat & $8.35-8.40$ & -7.4 & $54^{\circ} 8^{\prime} 36^{\prime \prime}$ & $15^{\circ} 20^{\prime} 9^{\prime \prime}$ & $9590 \pm 290$ & $\begin{array}{c}11294-11302(0.4 \%) \\
10490-11288(66.8 \%) \\
10438-10456(0.9 \%)\end{array}$ & Gd-30177 \\
\hline 23 & Trz28/103 & Trz28 & organic silt & $9.10-9.20$ & -8.1 & $54^{\circ} 8^{\prime} 36^{\prime \prime}$ & $15^{\circ} 20^{\prime} 9^{\prime \prime}$ & $11380 \pm 60$ & $13181-13316(68.2 \%)$ & Poz-33383 \\
\hline 24 & Trz28/113 & Trz28 & organic silt & $\begin{array}{l}9.90- \\
10.00\end{array}$ & -8.9 & $54^{\circ} 8^{\prime} 36^{\prime \prime}$ & $15^{\circ} 20^{\prime} 9^{\prime \prime}$ & $11120 \pm 60$ & $12926-13108(68.2 \%)$ & Poz-33384 \\
\hline 25 & Trz29/10 & Trz29 & peat & $1.1-1.15$ & 0.4 & $54^{\circ} 8^{\prime} 36^{\prime \prime}$ & $15^{\circ} 18^{\prime} 59^{\prime \prime}$ & $1700 \pm 50$ & $\begin{array}{l}1654-1691(19.6 \%) \\
1547-1628(48.6 \%)\end{array}$ & GdS-766 \\
\hline 26 & Trz29/19 & $\operatorname{Trz} 29$ & peat & $1.85-1.90$ & $-0,40$ & $54^{\circ} 8^{\prime} 36^{\prime \prime}$ & $15^{\circ} 18^{\prime} 59^{\prime \prime}$ & $5630 \pm 50$ & $\begin{array}{l}6392-6466(43.1 \%) \\
6321-6371(25.1 \%)\end{array}$ & Gd-11985 \\
\hline 28 & Trz29/57 & $\operatorname{Trz} 29$ & peat & $5.7-5.75$ & $-4,20$ & $54^{\circ} 8^{\prime} 36^{\prime \prime}$ & $15^{\circ} 18^{\prime} 59^{\prime \prime}$ & $7320 \pm 120$ & $\begin{array}{l}8260-8300(9.0 \%) \\
8010-8212(59.2 \%)\end{array}$ & GdS-767 \\
\hline
\end{tabular}




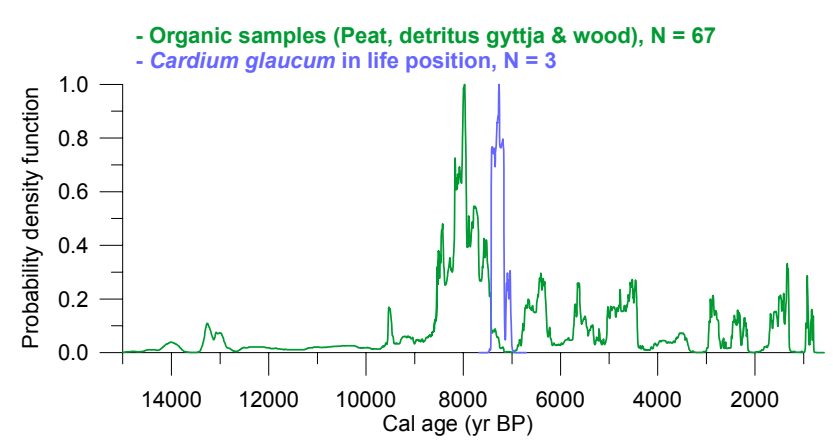

Fig. 6. Frequency distributions of radiocarbon dates of organic and shell samples on calendar time scale. The curves were constructed by summing up probability distribution of individual dates (Option "Sum" in OxCal programme) (B area).

around $-5.5 \mathrm{~m}$ amsl, a younger series of marine sediments can be found containing relatively large representations of malacofauna marine shells, especially Cardium glaucum and Macoma balthica.

The age of Cardium glaucum shells, determining the second period of accumulation of marine sediments, occurred between 7370 and the $7150 \mathrm{cal}$ BP. The oldest peat layer lying on these sediments does not exceed an age of $6740 \mathrm{cal} \mathrm{BP}$.

Based on data from the profile (Fig. 7) and other locations, it can be suggested that this area experienced two brief episodes of marine sedimentation which lasted for about 180 years (the earlier one) and from about 200 to max. 600 years (the later one).

\section{DISCUSSION AND CONCLUSIONS}

Comparing the periods of marine deposit formation in the aforementioned sedimentary basins, it may be noted that the ingression of Baltic waters associated with the socalled Littorina transgression was not synchronous in these two areas. It started earlier in the area of Mrzeżyno (Fig. 8) around 8300-8200 cal BP. Deposits that developed during that first phase contained few fragments of shells, therefore it is possible that they represent the facies of washover fans forming during heavy storms on the landward side of the spit, combined with a surge of waters on the southern coast of the Baltic sea. Such fossil washover fans are known from many sections of sandbar coasts including the area of Karwia (Gajewski et al., 2004) and on the southern coast of the Hel Spit (Tomczak, 1999). This interpretation is also suggested by the short-term accumulation of these deposits, not greater than 180 years. Given that their age was determined only indirectly, it cannot be excluded that they were accumulated during an even shorter period.

Analyzing the most recent literature on the ingression of the sea in the South Baltic area, clear signs can be found of marine ingression about 8300-8200 cal BP, for example in the Gardno-Łeba Costal Plain (Rotnicki,
2009). On the nearby Lithuanian coast, the first ingression occurred significantly earlier, about 8900 cal BP (Bitinas and Damušyte, 2004).

The second phase of ingression in the Mrzeżyno area started about 7300 years cal BP. These younger marine sediments already contained a considerable number of malacofauna marine shells, especially Cardium glaucum, often found in intravital position. This phase of ingression was also rather short, ending $6700 \mathrm{cal} \mathrm{BP}$ as is evidenced by the following geological facts:

- the presence of the oldest peat deposits about $6700 \mathrm{cal}$ $\mathrm{BP}$, on the marine sands,

- the presence of fluvial sediments, older than $6600 \mathrm{cal}$

$\mathrm{BP}$, inserted in the marine sands

In the area of the Szczecin Lagoon and the Świna Sandbar (Fig. 9), the oldest marine ingression could not have begun earlier than 7360 cal BP (Borówka et al., $2002,2005)$, and therefore more or less at the same time as the second phase in the area of Mrzeżyno. The quite clear trace is a considerably thick series of sands with numerous shells representing the marine and brackishmarine environments (Woziński et al., 2003), including the shells in life position of Cardium glaucum which make it possible to identify the age and the rate of accumulation of marine sands (Borówka et al., 2009). Geological documentation of the Szczecin Lagoon suggests that the accumulation of marine sediments continued uninterrupted until about 3800 cal BP.

That second phase of sea ingression occurred during the period of a sea level rise on the Gardno- $€$ eba Coastal Plain (Rotnicki, 2009). It also coincided with a distinct

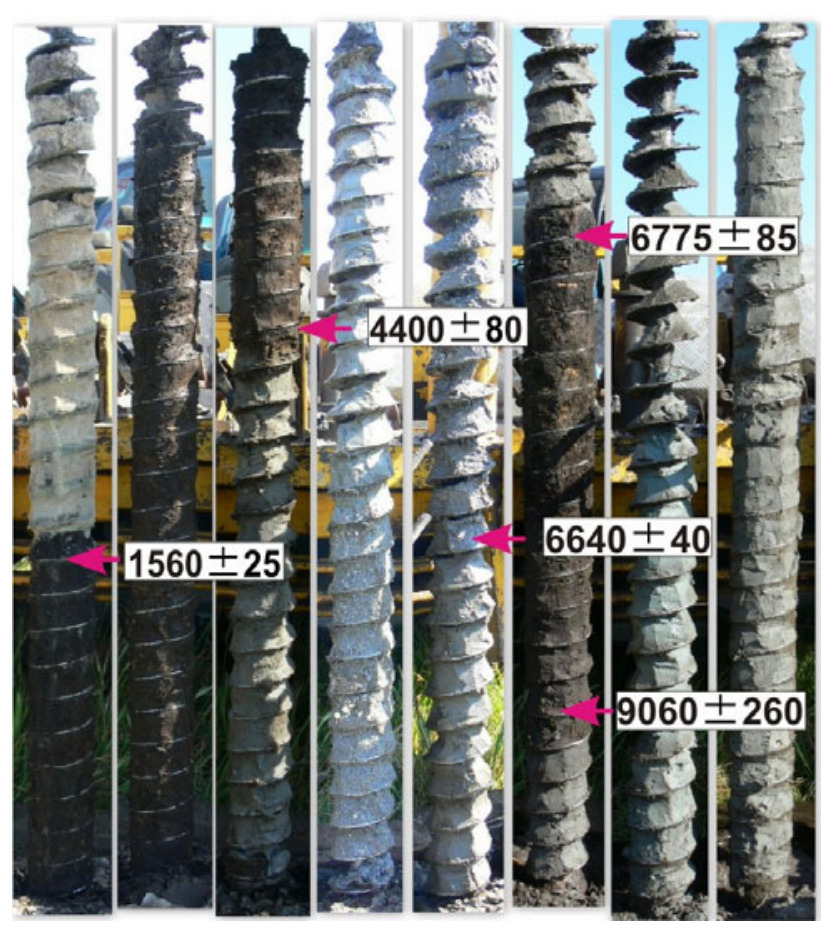

Fig. 7. Sediment cores 


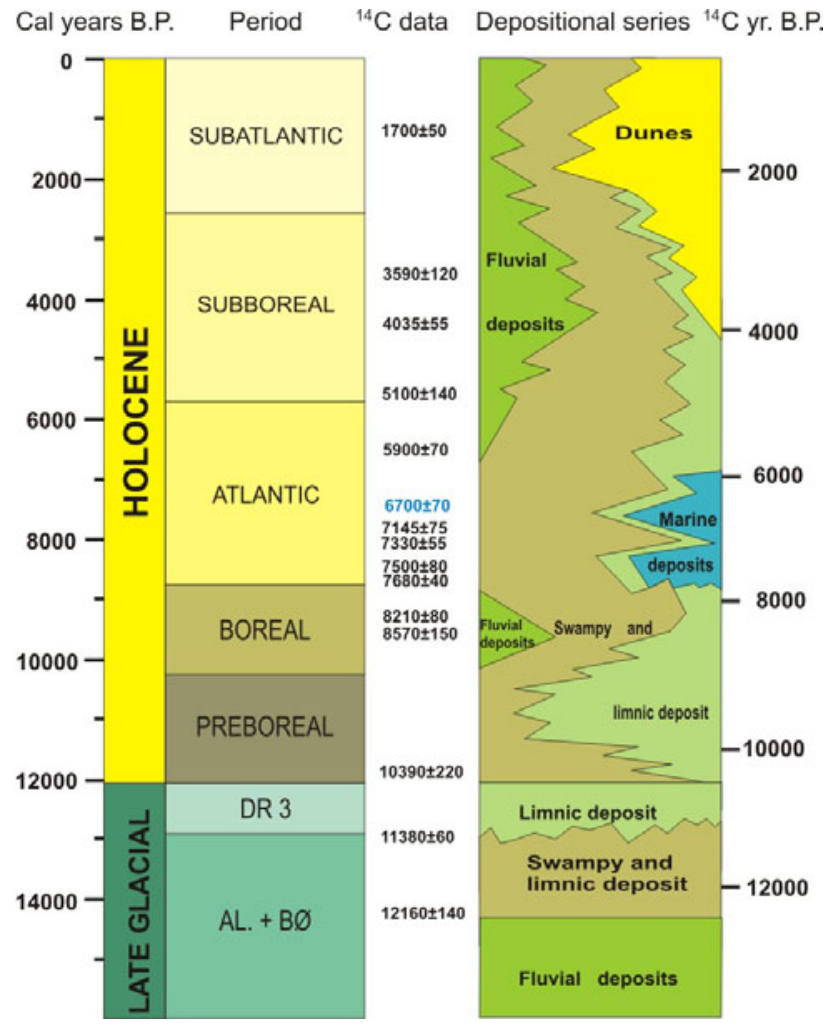

Fig. 8. Stratigraphy of the Mrzeżyno (B area)

rise in the level of the Baltic on the Lithuanian coast (Bitinas and Damušyte, 2004). In Lithuania, similar to the area of Mrzeżyno, that phase of sea level rise was relatively short and followed by a very clear regression which in the vicinity of Mrzeżyno resulted in the insertion of Rega river into marine sediments. Within the lagoon itself there is no clear evidence of subsequent sea regression. This is probably due to the fact that the marine works in the Szczecin Lagoon formed at a much lower ordinate compared to the area of Mrzeżyno.

It should also be noted that ingressions appeared earlier in the Gardno-Łeba Coastal Plain and in the area of Mrzeżyno, compared to Szczecin Lagoon. This was probably caused by the different bathymetric situation of the bottom of the Baltic Sea in the area adjacent to the Świna Sandbar and the Pomeranian Bay, where shallow waters occur at a considerable distance from the presentday shore, reaching just behind the northern border of the Oderbank Plateau. During the marine ingression, combined with the rising sea level, at the bottom of the Pomeranian Bay to the shore was significantly longer in comparison with the area of Mrzeżyno and the zone adjacent to the Gardno-Łeba Coastal Plain. This distance must have taken much more time to cover, hence a short-term ingression could only be observed where the $20 \mathrm{~m}$ isobath is located near the modern coastline.

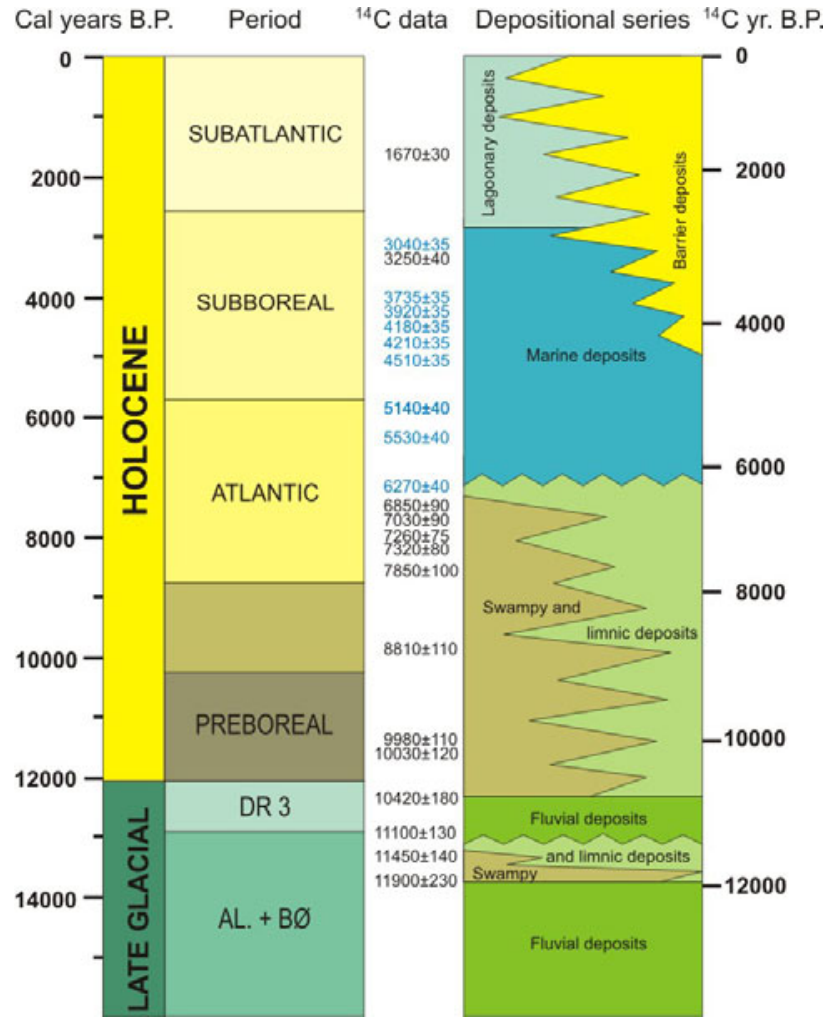

Fig. 9. Stratigraphy of the Szczecin Bay (A area)

\section{ACKNOWLEDGEMENTS}

The study was partially supported by the Polish Committee for Scientific Research grant No 6PO4E 002 16, No 2PO4E 05030, No 2PO4E 04129, No N N306 222137. The authors are grateful to Dr Danuta Michczyńska for help in preparing figures and tables.

\section{REFERENCES}

Bitinas A, Damusyte A, 2004. Littorina sea at the Lithuanian maritime region. Polish geological institute Special papers 11: 37-46.

Borówka RK, Witkowski A, Tomkowiak J, Olas M, Gusar K and Liszkiewicz E, 1999. Stratygraficzna i przestrzenna zmienność litologiczna i geochemiczna osadow wypełniających Zalew Szczeciński i baseny przyległe. (Stratigraphic and spatial litological and geochemical variability of sediments In the Szczecin Haff and adjacent basins) In: Borówka RK, Młynarczyk Z and Wojciechowski A, eds., Ewolucja geosystemow nadmorskich Poludniowego Battyku. Bogucki Wydawnictwo Naukowe. PoznańSzczecin: 55-62. (in Polish).

Borówka RK, Latałowa M, Osadczuk A, Święta J and Witkowski A, 2002. Palaeogeography and palaeoecology of Szczecin Lagoon. Greifswalder Geographische Arbeiten 27(C4): 107-113.

Borówka RK, Osadczuk A, Witkowski A, Wawrzyniak-Wydrowska B and Duda T, 2005. Late Glacial and Holocene depositional history in the eastern part of the Szczecin Lagoon (Great Lagoon) basin NW Poland. Quaternary International 130(1): 87-96, DOI 10.1016/j.quaint.2004.04.034.

Borówka RK, Witkowski A, Latałowa M and Skowronek A, 2009. Sedimentation of a marine deposit in the Szczecin Bay during the Littorina transgression. In: Witkowski A, Harff $\mathrm{J}$ and Isemer H-J, 
eds., International Conference on Climate Change - The environmental and socio-economic response in the southern Baltic region. Conference Proceedings, BALTEX, Publication No. 42: 8 .

Bronk Ramsey C, 2009. Bayesian analysis of radiocarbon dates. Radiocarbon 51(1): 337-360.

Cedro B, 2003. Postglacjalne i holoceńskie osady fluwialne, jeziornobagienne i morskie w dolinie Regi koło Mrzeżyna (Postglacial and Holocene sediments: fluvial, swampy and maritime in Rega valley near Mrzeżyno). In: Borówka RK and Witkowski A, eds., Człowiek i środowisko przyrodnicze Pomorza Zachodniego, Oficyna IN PLUS Szczecin: 47-49 (in Polish).

Cedro B, 2005. Wstepne uwagi o wieku osadów wypełniajacych doline Regi w rejonie Łobza i jej ujściowym odcinku w rejonie Mrzeżyna (Preliminary notes about age of sediments in Rega valley in vicinity of Łobez and in estuary sector in Mrzeżyno region). In: Borówka RK, ed., Plejstoceńskie i holoceńskie przemiany środowiska przyrodniczego Polski. Wybrane aspekty. Oficyna InPlus: 13-16 (in Polish).

Cedro B, 2008. Zapis zmian poziomu południowego Bałtyku w okolicy Mrzeżyna. (Recorded changes in the level of the southern Baltic Sea in the area Mrzeżyno). Holoceńskie przemiany wybrzeży $i$ wód poludniowego Bałtyku - przyczyny, uwarunkowania i skutki. Smołdzino 6-9.05.2008: 47 (in Polish).

Chrono Marine Reservoir Database, 2005 WEB site: $<$ http://intcal.qub.ac.uk/marine/>. Accessed 2010 August 14.

Gajewski L, Gajewski Ł, Rudowski S and Stachowiak A, 2004. The relief of the offshore sea bottom at the Karwia-Chałupy, Polish Baltic Coast. Proceedings of the Conference "Rapid transgressions into semi-enclosed basins". Polish Geological Institute, Special Papers. 11: 91-94.

Karczewski A, 1968. Wpływ recesji lobu Odry na powstanie i rozwój sieci dolinnej Pojezierza Myśliborskiego i Niziny Szczecińskiej. (Influence of the Odra lobe recession upon the origin and devel opment of the valley net of Myślibórz Lakeland and Szczecin Lowlan). Prace Komisji Geograficzno-Geologicznej Poznańskiego Towarzystwa Przyjaciót Nauk 8 (3): 1-106 (in Polish).

OxCal - online radiocarbon calibration, 1999 ,WEB site: < https://c14.arch.ox.ac.uk/> Accessed 2010August 14

Reimer PJ, Baillie MGL, Bard E, Bayliss A, Beck JW, Blackwell PG, Bronk Ramsey C, Buck CE, Burr G, Edwards RL, Friedrich M, Grootes PM, Guilderson TP, Hajdas I, Heaton TJ, Hogg AG, Hughen KA, Kraiser KF, Kromer B, McCormac FG, Manning S, Reimer RW, Richards DA, Southon JR, Talamo S, Turney CSM, van der Plicht J, Weyhenmeyer CE, 2009. IntCal09 and Marine09 radiocarbon age calibration curves, $0-50,000$ years cal BP. Radiocarbon 51: 1111-1150.

Rotnicki K, 2009. Identyfikacja, wiek i przyczyny holoceńskich ingresji i regresji Bałtyku na polskim wybrzeżu środkowym. (Ideatyfication, age and causes of the Holocene ingressions and regressions of the Baltic on the Polish Middle Coast). Wydawnictwo Slowińskiego Parku Narodowego: 1-100 (in Polish, English summary).

Tomczak A, 1999. Storm Overflows in the Western Part of the Hel Peninsula on Maps from the Years 1694, 1818 and 1844, and Its Contemporary Relief. In: R Gołębiewski, ed., Peribalticum. GTN, Gdańsk: 89-98.

Wołowicz M, 1991. Geograficzne zróżnicowanie populacji Cardium glaucum Bruguiere (Bivalvia). Hipotezy dotyczące pochodzenia gatunku oraz dróg migracji. (The geographic diversity of Cardium glaucum Bruguiere (Bivalvia). Hypotheses on the origin of species and migration routes). Zeszyty Naukowe UG: 1-157 (in Polish).

Woziński R, Wawrzyniak-Wydrowska B and Borówka RK, 2003. The subfossil malakofauna from the Holocene deposits of the Szczecin Lagoon. In: Borówka RK andWitkowski A, eds., Człowiek i środowisko przyrodnicze Pomorza Zachodniego. Oficyna IN PLUS Szczecin:113-118 (in Polish). 